\title{
Prototype Milli Gauss Meter Using Giant Magnetoimpedance Effect in Self Biased Amorphous Ribbon
}

\author{
Pratap Kollu ${ }^{1}$, Seok Soo Yoon ${ }^{2 *}$, Gun Woo Kim ${ }^{1,2}$, C. S. Angani ${ }^{1}$, and Cheol Gi Kim ${ }^{1 *}$ \\ ${ }^{1}$ Department of Materials Science and Engineering, Chungnam National University, Daejeon 305-764, Korea \\ ${ }^{2}$ Department of Physics, Andong National University, Kyoungsangbuk-do 760-749, Korea
}

(Received 6 September 2010, Received in final form 28 September 2010, Accepted 29 September 2010)

\begin{abstract}
In our present work, we developed a GMI (giant magnetoimpedance) sensor system to detect magnetic fields in the milli gauss range based on the asymmetric magnetoimpedance (AGMI) effect in Co-based amorphous ribbon with self bias field produced by field-annealing in open air. The system comprises magnetoimpedance sensor probe, signal conditioning circuits, A/D converter, USB controller, notebook computer, and program for measurement and display. Sensor probe was constructed by wire-bonding the cobalt based amorphous ribbon with dimensions $10 \mathrm{~mm} \times 1 \mathrm{~mm} \times 20 \mu \mathrm{m}$ on a printed circuit board. Negative feedback was used to remove the hysteresis and temperature dependence and to increase the linearity of the system. Sensitivity of the milli gauss meter was $0.3 \mathrm{~V} / \mathrm{Oe}$ and the magnetic field resolution and environmental noise level were less than $0.01 \mathrm{Oe}$ and 2 mOe, respectively, in an unshielded room.
\end{abstract}

Keywords : milli guass meter, amorphous soft magnetic ribbon, magnetic sensor, magnetoimpedance, negative feedback

\section{Introduction}

In the today's world, micro-sized magnetic sensors with high sensitivity, quick response, and low cost are greatly required. GMI sensors have magnetic field resolutions comparable with flux-gate sensors, without a need for exciting and sensing coils [1]. Additionally, GMI sensors have been found to be more field sensitive than the present giant magneto-resistance (GMR) sensors $[2,3]$. The GMR materials generally involve large fields to obtain a response of a few percent of resistance change, whereas the GMI materials can detect very small magnetic fields, producing a few hundred percent changes in the impedance. The GMI sensitivity observed in the amorphous microwires was in the range of $10 \sim 100 \% / \mathrm{Oe}$ at $\mathrm{MHz}$ frequencies [4]. This sensitivity is comparatively very high to the GMR materials. Furthermore, another advantage of the GMI effect is that it does not exhibit hysteresis, as is the case with GMR materials; hysteresis is not desirable for most of the sensor applications since it causes the loss of power as well as heating of the device. Extensive research

\section{*Corresponding authors:}

Tel: +82-54-820-5450, Fax: +82-54-823-1628, e-mail: yoon@andong.ac.kr Tel: +82-42-821-6632, Fax: +82-42-822-6272, e-mail: cgkim@cnu.ac.kr has been done in amorphous materials in the form of wires and ribbons, as they show excellent GMI response due to the very soft magnetic properties that they exhibit [5].

Developing high resolution magnetic sensors utilizing the GMI effect is the latest trend in the industry and research community since the potential applications were envisaged by Kaneo Mohri [6]. Since then, this group pioneered the research in GMI sensors using C-MOS IC multivibrator technique. They constructed the magnetic field detection sensors using $\mathrm{FeCoSiB}$ amorphous microwire. Two wires with $30 \mu \mathrm{m}$ diameters and $1 \sim 2 \mathrm{~mm}$ lengths have been used as sensor heads. Asymmetric behavior in the output is obtained by applying DC bias field by using two coils. Negative feedback is employed for removing the noise and temperature dependence; two more coils are used for this purpose. A stable GMI sensor with field detection resolution of $10^{-6}$ Oe was made [7].

H. Hauser et al. also constructed a magnetic field sensor using GMI in CoSiBNd wire with $70 \mu \mathrm{m}$ length and $30 \mu \mathrm{m}$ diameter. This sensor was fabricated using the frequency modulation technique platform. A magnetic field resolution of $100 \mathrm{nT}$ was achieved [8]. G. P. Jian et al. constructed a magnetometer using amorphous microwires [9]. The sensor possesses sensitivity of $18 \mu \mathrm{V} / \mu \mathrm{T}$ and 
magnetic field resolution of $100 \mathrm{nT}$. Yabukami et al. used $\mathrm{CoNbZr}$ thin film as head element and achieved an extremely high resolution magnetic field detection of 1.7 $\times 10^{-8} \mathrm{Oe}$ at $500 \mathrm{kHz}$ [10].

The only commercially available magnetic sensor based on the GMI effect is produced from the Aichi MicroIntelligent (Aichi MI) company in Japan [11]. It has magnetic field resolution of $10 \mathrm{nT}$.

The idea to develop magnetic field detection sensors by using ribbon as the sensing head began with the discovery of asymmetric GMI in the field-annealed $\mathrm{Co}_{66} \mathrm{Fe}_{4} \mathrm{Si}_{15} \mathrm{~B}_{15}$ ribbon in open air. We reported that the asymmetric GMI effect in the field-annealed ribbon due to the bias field produced by hard magnetic layer formed near the surface during annealing $[5,12]$.

This asymmetric profile is very important for linear field magnetic sensors. Mohri et al. used two DC bias coils to achieve this asymmetry for two sensing elements, which require more power and generates heat that degrades the performance of the sensor [6]. Our field-annealed ribbon exhibits asymmetric profile due to the self bias field, so we do not require any additional circuit to get asymmetric behavior. We reported fundamental circuit to develop magnetic sensor based on the asymmetric GMI effect of the self biased ribbon and improved circuit for negative feedback to increase linearity and to avoid temperature dependence $[2,3]$. In this work, we developed the prototype gauss meter based on the asymmetric GMI effect of the self biased amorphous ribbon and we report its performance.

\section{Development of Gauss Meter}

\subsection{GMI Sensor Probe}

The asymmetric GMI sensor head with dimensions of 1 $\mathrm{mm} \times 10 \mathrm{~mm} \times 20 \mu \mathrm{m}$ were fabricated by field annealing the $\mathrm{Co}_{66} \mathrm{Fe}_{4} \mathrm{Si}_{15} \mathrm{~B}_{15}$ ribbon using our patented techniques for producing self bias field [Kor. Patent No. 10-0383564, (2003)]. For contacting the sensor head with current and voltage lines, gold electrodes were deposited on both ends of the head using electrodeposition. The sample was masked in middle with a $6 \mathrm{~mm}$ kapton film. Hence, the 2 $\mathrm{mm}$ edges were opened on both sides. The chemical solutions that we used for electrodeposition were purchased from SmeKorea Company [13].

A printed circuit board (pcb) was designed for placing the sensor head and electrode contact. Four lines were present in the pcb, two of them were for current and voltage and other two for connecting the feedback coil. The contact area was made of gold on the pcb. For contacting the sensor head with pcb, ultrasonic wire bonder was

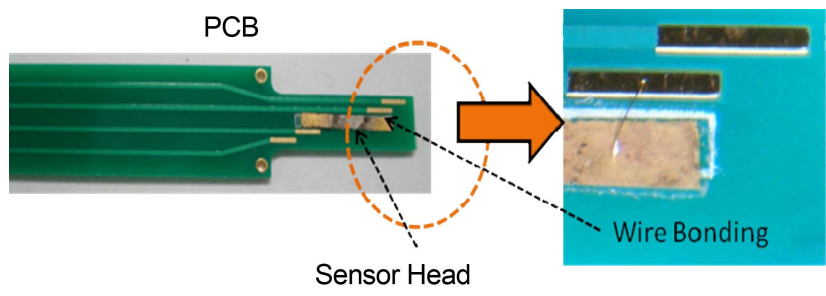

Fig. 1. Photo of the sensor probe showing wire bonded sensor head of amorphous ribbon.

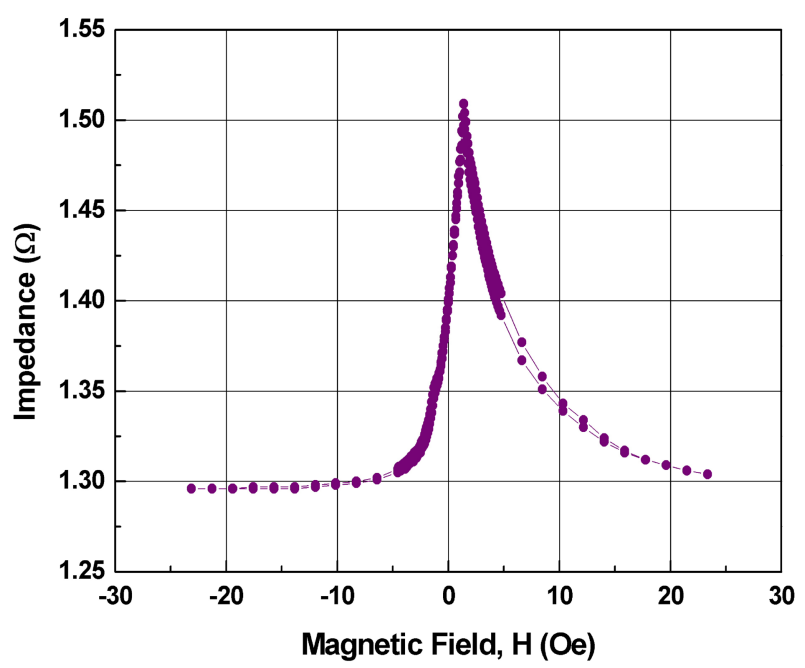

Fig. 2. Impedance Vs. magnetic field measured for $10 \mathrm{~mm}$ asymmetric GMI ribbon at $100 \mathrm{kHz}$.

used. Fig. 1 shows the photo of GMI sensor probe fabricated by using the above method.

Fig. 2 shows the GMI profile of the sensor head measured with a HP4192A impedance analyzer at $100 \mathrm{kHz}$. This shows typical asymmetric profile of the self biased amorphous ribbon by field-annealing, and there is no change in the GMI profile due to electrodeposition and wire bonding for probe.

\subsection{Complete system}

We constructed the GMI sensor system for gauss meter utilizing the asymmetric GMI probe. Fig. 3 shows the block diagram of the gauss meter system. The system comprises GMI sensor probe, signal conditioning with battery power supply, A/D converter with USB controller, notebook computer, and program for measurement and display. The core part in the gauss meter system is the signal conditioning circuit. Fig. 4 shows the block diagram of the signal conditioning circuit, which is based on the circuit developed in our previous works $[2,3]$. This circuit consists of an oscillator generating $100 \mathrm{kHz}$ sine wave; this signal passes through a voltage to current converter. 


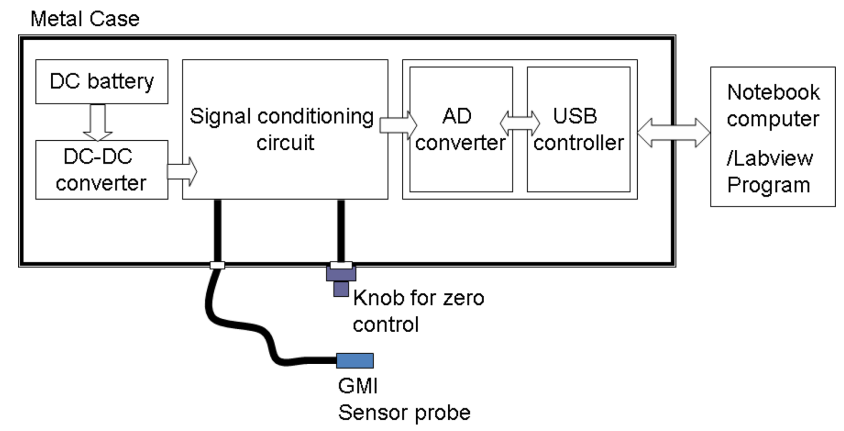

Fig. 3. Block diagram of the gauss meter.

The converted AC current with constant amplitude of 10 $\mathrm{mA}$ is supplied to the GMI sensor head. The induced voltage across the sensor head is picked up by differential amplifier. In order to get DC output voltage proportional to the amplitude of in-phase component of the induced $\mathrm{AC}$ voltage with oscillator voltage, phase sensitive detection method(lock-in amplifier method) is adopted through the multiplier and low pass filter circuit. The offset control circuit is added to the signal conditioning circuit for controlling the DC output voltage to zero in the absence of magnetic field. The final DC output voltage is applied to negative feedback coil that is wound around the sensor head. This negative feedback improves the linearity and reduces the hysteresis and temperature dependence, and causes the final DC output voltage to linearly depend on the applied magnetic field when a condition is fulfilled. The principle and condition of the negative feedback were explained in detail in our previous paper [3].

Noise was observed due to $60 \mathrm{~Hz}$ electrical line and $100 \mathrm{kHz}$ oscillator, these components were observed in the output. $12 \mathrm{~V}$ battery was used to eliminate the electrical noise and $1 \mathrm{kHz}$ LPF (low pass filter) was used to remove the $100 \mathrm{kHz}$ noise component at the output.

\section{Performance of Gauss Meter}

In order to test the performance of the developed gauss meter system, we measured the change of final DC output

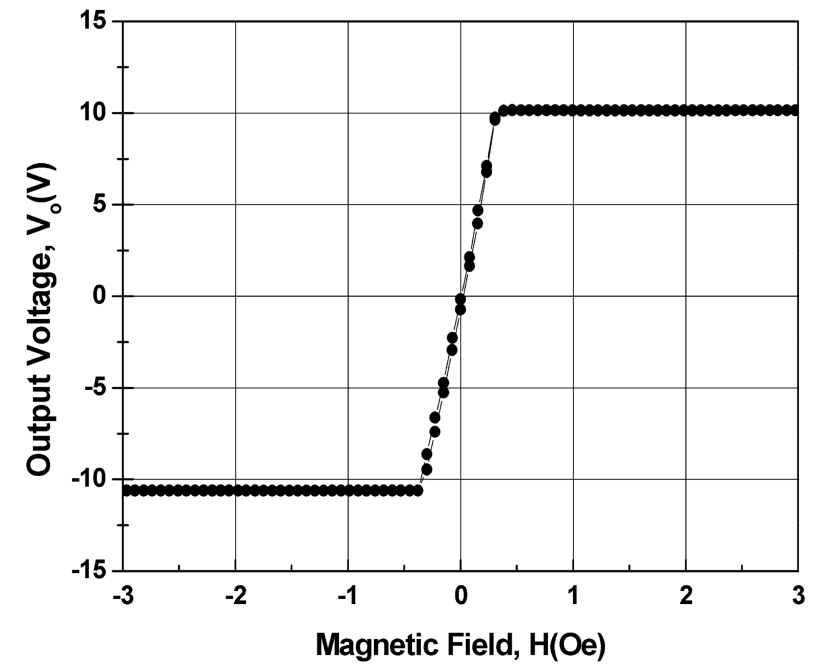

Fig. 5. GMI profile of the sensor without negative feedback.

voltage $V_{o}$ with applied magnetic field $H$ with GMI sensor probe in Helmholtz coil. Fig. 5 shows a response curve for $H$ of the gauss meter without negative feedback. The curve shows almost linear response with slope of $30 \mathrm{~V} /$ Oe, which can be expressed by the following relation:

$$
V_{o}=A H, A=30 \mathrm{~V} / \mathrm{Oe} \text {, }
$$

where $A$ is the transfer constant of the gauss meter system which converts a magnetic field to output voltage.

The transfer constant $A$ depends on the slope of GMI curve that is shown in Fig. 2. This slope depends on the gains of amplifiers in the signal conditioning circuit. We maximized $A$ by controlling gains in the range, such that the $\mathrm{AC}$ voltage signals are not distorted by saturation. This is because highest possible value of $A$ is required to get good linear response with negligible hysteresis and temperature dependence by using negative feedback.

Fig. 6 shows the response curve for $H$ when the output voltage is applied to negative feedback coil. The curve shows linear response with linearity error less than $0.2 \%$ in the dynamic range between -2 Oe $\sim 2$ Oe. The gauss meter system shows sensitivity of $0.3 \mathrm{~V} / \mathrm{Oe}$.

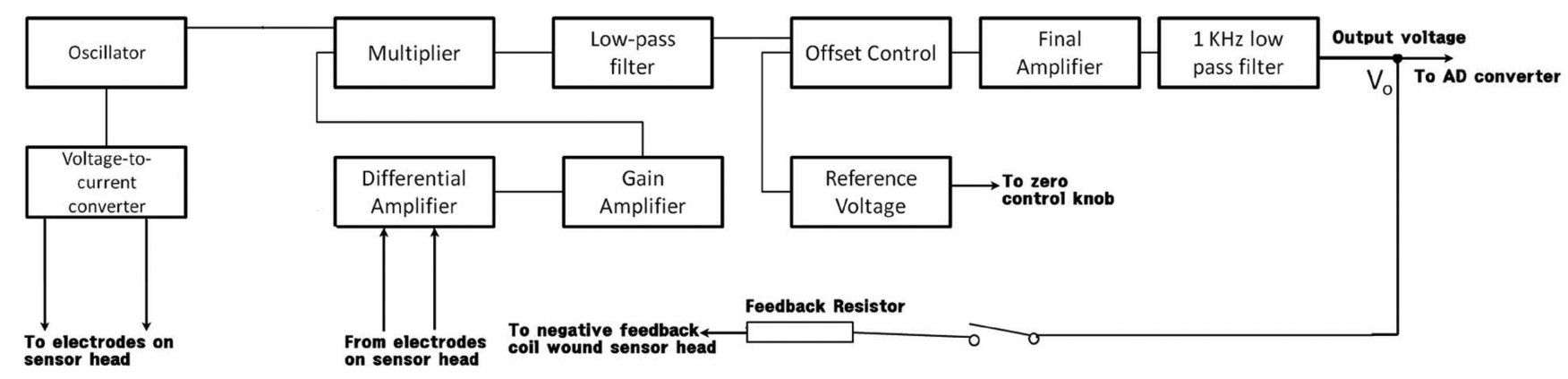

Fig. 4. Block diagram of the signal conditioning circuit. 


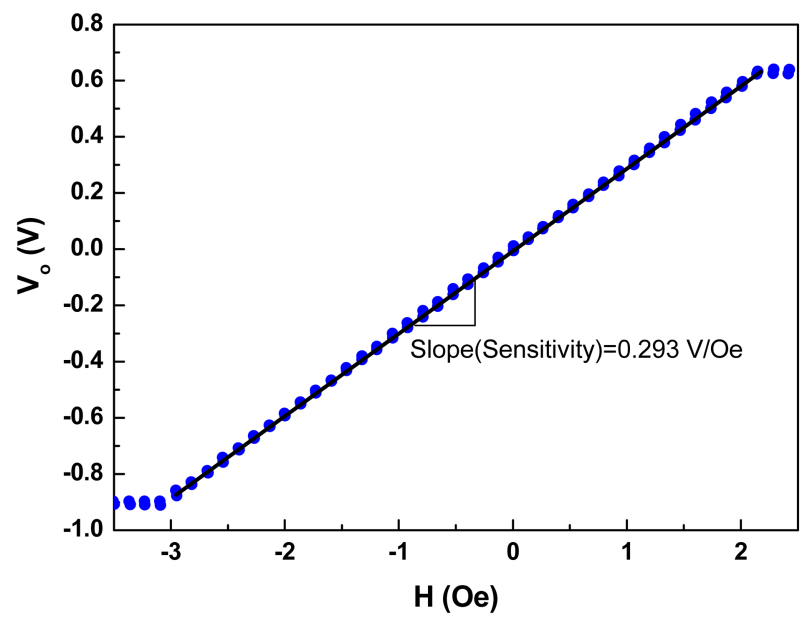

Fig. 6. GMI profile of the sensor with negative feedback.

Since the feedback solenoid coil wound sensor head has number of turns $N=80$ and length $l=12 \mathrm{~mm}$ and connected to the output voltage through the feedback resistance $R_{f}=24.2 \Omega$, the output voltage $V_{\mathrm{o}}$ is converted to feedback magnetic field $H_{f}$ with the transfer constant $S$ by the relation:

$$
H_{f}=S V_{o}, S=4 \pi \times 10^{-3} \frac{N}{R_{f} l}=3.46 \mathrm{Oe} / \mathrm{V},
$$

The $V_{o}$ of the gauss meter system follows the relation [3]

$$
V_{o}=\frac{A}{1+A S} H \text {. }
$$

Since $A S=103.8$ in the present gauss meter system, we can get the approximation:

$$
V_{o} \approx \frac{1}{S} H, \text { if } \mathrm{AS}>>1 \text {. }
$$

The slope of 0.293 V/Oe in Fig. 6 shows good agreement with the $1 / S=0.289$. This means that the $V_{o}$ with negative feedback linearly depends on $H$ and the sensitivity depends only on the coil parameters, such as $N, l$, and $R_{f}$. Since these coil parameters have negligible temperature dependence in normal operation range of gauss meter, we can avoid temperature calibration problem.

Other important performance characteristics of gauss meter system are resolution and noise level. In order to test these performances, change in the output voltage $V_{o}$ is monitored by altering the magnetic field in uniform steps by using HP 6632A programmable power supply. Fig. 7 shows the change of $V_{o}$ for the 0.01 Oe step which is the minimum controllable step in our laboratory. The change of $0.01 \mathrm{Oe}(1 \mu \mathrm{T})$ in the magnetic field is clearly detectable and environmental noise level is about $2 \mathrm{mOe}$. We

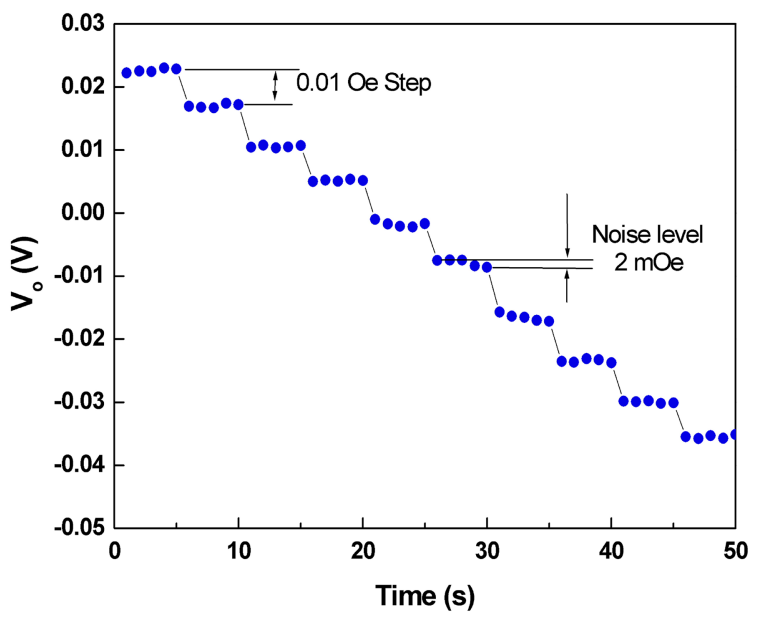

Fig. 7. Resolution check by decreasing the magnetic field by steps of 0.01 Oe.

measured the resolution and noise level in the room which is not electromagnetically shielded. The gauss meter is expected to possess high resolution and low noise level, if the experiment is performed in magnetic shielding chamber.

\section{Conclusion}

A complete system for detecting the magnetic fields in milligauss range was successfully developed using self biased GMI. It was successfully developed and its performance was checked. The sensitivity was improved to $\sim 0.3 \mathrm{Oe} / \mathrm{V}$ with negative feedback system. Electrical line noise was removed by using a $12 \mathrm{~V}$ battery and the 100 $\mathrm{kHz}$ component was eliminated by the end-filter. The resolution of the gauss meter was $1 \mu \mathrm{T}$.

\section{Acknowledgements}

This research was financially supported by a grant to MEMS Research Center for National Defense funded by Defense Acquisition Program Administration.

\section{References}

[1] T. Morikawa, Y. Nishibe, H. Yamadera, Y. Nanomura, M. Takeuchi, and Y. Taga, IEEE Trans. Magn. 32, 5 (1996).

[2] P. Kollu, L. Jin, K. W. Kim, S. S. Yoon, and C. G. Kim, Appl. Phys. A 90, 533 (2008).

[3] S. S. Yoon, P. Kollu, K. W. Kim, Y. Cha, D. Y. Kim, and C. G. Kim, IEEE Trans. Magn. 45, 2727 (2009).

[4] P. Delooze P. Delooze, L. V. Panina, D. J. Mapps, K. Ueno, and H. Sano, IEEE Trans. Magn. 39, 3307 (2003).

[5] C. G. Kim, K. J. Jang, H. C. Kim, and S. S. Yoon, J. 
Appl. Phys. 85, 5447 (1999).

[6] K. Mohri, T. Uchiyama, and L. V. Panina, Sens Actuators A 59, 1 (1997).

[7] T. Kanno, K. Mohri, T. Yagi, T. Uchiyama, and L. P. Shen, IEEE Trans. Magn. 22, 3358 (1997).

[8] H. Hauser, R. Steindl, C. Hausleitner, A. Pohl, and J. Nicolics, IEEE Trans. Instrum. Meas. 49, 648 (2000).

[9] G. P. Jian and T. G. Qing, Journal of Beijing Institute of Machinery 25, 1 (2010). http://d.wanfangdata.com.cn/
Periodical_bjjxgyxyxb201001013.aspx

[10] S. Yabukami, H. Mawatari, N. Horikoshi, Y. Murayama, T. Ozawa, and K. Ishiyama, J. Magn. Magn. Mater. 290, 1318 (2005).

[11] http://www.aichi-mi.com

[12] C. G. Kim, K. J. Jang, D. Y. Kim, and S. S. Yoon, Appl. Phys. Lett. 75, 2114 (1999).

[13] http://www.smekorea.com 